Revista de la red interuniversitaria de estudios sobre las literaturas rioplatenses contemporáneas en Francia

$11 \mid 2014$

De niños e infancias

\title{
El despertar del padre : escritura e infancia en Domingo Faustino Sarmiento
}

\section{Alejandra Josiowicz}

\section{OpenEdition}

\section{Journals}

Edición electrónica

URL: http://journals.openedition.org/lirico/1775

DOI: $10.4000 /$ lirico. 1775

ISSN: 2262-8339

Editor

Réseau interuniversitaire d'étude des littératures contemporaines du Río de la Plata

\section{Referencia electrónica}

Alejandra Josiowicz, «El despertar del padre : escritura e infancia en Domingo Faustino Sarmiento »,

Cuadernos LIRICO [En línea], 11 | 2014, Puesto en línea el 01 diciembre 2014, consultado el 19 abril 2019. URL : http://journals.openedition.org/lirico/1775; DOI : 10.4000/lirico.1775

Este documento fue generado automáticamente el 19 abril 2019.

\section{cc) $(1) \odot$}

Cuadernos LIRICO está distribuido bajo una Licencia Creative Commons Atribución-NoComercialSinDerivar 4.0 Internacional. 


\title{
El despertar del padre : escritura e infancia en Domingo Faustino Sarmiento
}

\author{
Alejandra Josiowicz
}

1 Este ensayo se propone reflexionar sobre la escena de la muerte del niño en La vida de Dominguito, ${ }^{1}$ de Domingo Faustino Sarmiento, en un sentido doble: por un lado, en su relación con el lenguaje, como evento que recupera y pone en escena el acceso a la letra, y, por otro, como experiencia traumática, que establece una discontinuidad fundamental en la experiencia del padre. La muerte del hijo adoptivo de Sarmiento, su único sucesor, es abordada en un plano autobiográfico y del significante poético ; en tanto trauma, dicho evento se liga a un despertar de la subjetividad del padre, a la vez que a un desvío o interrupción en la relación entre el sujeto, el lenguaje y la mortalidad. Se postula que la infancia aparece en el texto en tres niveles diferentes : en primer lugar, el niño figurado en el lenguaje, representado a través de la narración del recuerdo y la escritura autobiográfica ; en segundo, la letra del hijo incorporada en el texto a través del montaje documental; $y$, por último, la muerte del niño como emergencia del trauma en su irreducible otredad, aquello de lo cual el sujeto no puede dar cuenta, pero a lo que no puede dejar de responder en su carácter de superviviente o testigo. La vida de Dominguito, como texto sobre el despertar del padre a la otredad del hijo y encuentro con su mortalidad, responde a la necesidad ética y, a la vez, a la imposibilidad de narrar la escena traumática de la muerte del hijo.

\section{Escrituras del duelo}

Domingo Fidel Sarmiento, el único hijo que Sarmiento consideró su sucesor intelectual, nació en Chile en 1845 y fue adoptado luego de que, durante su exilio, Sarmiento se casara con su madre viuda. La crítica aún debate si Dominguito fue hijo biológico de Sarmiento, fruto de una unión extramatrimonial con la madre, Benita Martínez de Castro, o si simplemente fue adoptado luego de fallecido su padre. ${ }^{2}$ Lo cierto es que, en 1866 , cuando 
recibió la noticia de la muerte de Dominguito en la batalla de Curupaití, durante la Guerra del Paraguay, Sarmiento, que se desempeñaba en Washington como Ministro Plenipotenciario, quedó profundamente conmocionado: "La muerte de Dominguito" le escribió a Bartolomé Mitre en 1867, quien era comandante de su hijo, "tan malogrado, ha traído a mi espíritu un incurable descontento. ¡Qué de cadenas de desencantos! Habría vivido en él; mientras que ahora no sé dónde arrojar este pedazo de mi vida que me queda, pues ni aquí ni allá sé qué hacer con ella." (Fariña Nuñez, 1935 : 154) La muerte, como quiebre del tiempo histórico y discontinuidad fundamental, sacude a Sarmiento padre, que siente que su experiencia se ha vaciado de sentido y, como en el trabajo del duelo freudiano, se identifica con el objeto perdido (Freud, $1973: 244$ ). Como aparece en su carta a Mary Mann de 1866, Sarmiento recupera la escena de infancia como núcleo del duelo:

Tengo que conformarme, y ya estoy más resignado, aunque el recuerdo de sus gracias infantiles, sus juegos conmigo, me haga llorar más que la idea de su trágica y sangrienta muerte. No puedo recordarlo sino alegre y riendo y esto me hace sufrir más. Estos días estaré más tranquilo. Le agradezco su tierno interés y quedo su desconsolado amigo. (Sarmiento, $2000: 17$ )

3 No es la muerte épica del joven soldado de 20 años lo que aparece en el imaginario del sujeto del duelo, sino el recuerdo del niño pequeño, "alegre y riendo", entrelazado con el padre en el juego. La experiencia traumática lleva al padre, lejos de la historia pública, hacia la escena íntima, doméstica, de la infancia, encuentro con la presencia efímera del niño.

Mientras se encontraba en Washington, Sarmiento mantuvo un asiduo intercambio epistolar con Mary P. Mann, esposa de Horace Mann, quien le sugirió la lectura del poema elegíaco In memoriam (1849), de Alfred Tennyson, escrito a la muerte de su amigo Arthur Hallam, que ella misma consideraba un instrumento de duelo (Anderson Imbert, 1975 : 506). La vida de Dominguito emula a Tennyson en su propósito consolatorio : la escritura elegíaca, como inscripción de la pérdida del sujeto amado y del propio proceso del duelo, aparece dotada de un poder reparativo (Fuss, $2013: 5$ ). De hecho, el texto forma una serie junto a otros textos que, durante el Siglo XIX y XX, reflexionaron sobre el límite del lenguaje y de la propia experiencia ante la pérdida de la descendencia, la muerte del hijo : Pour un tombeau d'Anatole de Stéphane Mallarmé, escrito a la muerte de su hijo Anatole en 1879, así como "Threnody" y "Experience", de Ralph Waldo Emerson, escritos a la muerte de su hijo Waldo, en 1842 y 1844, son sólo algunos ejemplos. Así, en Pour un tombeau d'Anatole, de Mallarmé, el padre confronta, en la muerte del hijo, el vacío inherente en su imposibilidad de morir, así como en el desconocimiento del niño de su propia mortalidad ${ }^{3}$ . El poema funciona como puesta en escena de ese vacío : "sentir éclater / en nuit / le vide immense / produit par ce / qui serait sa vie / - parce qu'il ne / le sait pas- / qu'il est mort / éclair? /crise / douleur." (Fynsk, 2000: 86). Como se ve en el fragmento, el poema escribe la muerte del niño como crisis dolorosa, vacío "inmenso" que estalla. Se trata de un vacío doble : el de una potencia irrealizada, la vida futura del niño que no fue, y el del desconocimiento del niño de su propia muerte.

5 Ya entrado el Siglo XX, en Más allá del principio del placer (1920), Freud también parte de una escena autobiográfica para explorar la relación entre el sujeto y la muerte, y entre la experiencia traumática y la infancia. Freud parte de la observación del juego de su nieto, Ernst, con un trencito de madera para analizar el modo por el cual el sujeto lidia con la posible falta del objeto amado (la madre), a través de la repetición o reactualización de su 
partida, que llama, a partir de una de las exclamaciones del niño, fort-da (Freud, 1961 : 15). Esta escena autobiográfica, tal como la ha leído Jacques Derrida, implica el establecimiento de un contrato entre Freud y su legatario, que, al mismo tiempo, señala un legado teórico para el psicoanálisis (Freud, 1961 : 15). Este contrato a la vez teórico y autobiográfico se rompe cuando el legador, Freud, debe enfrentar el fallecimiento de los descendientes: de Sophie, su hija, en 1920, y, más tarde, de su otro nieto, Heinele, hermano de Ernst, a la edad de cinco años (Freud, 1961: 16). Como Sarmiento, Freud escribe, en una carta a Oscar Rie, sobre la muerte del descendiente-en este caso, el nieto -como experiencia traumática, ruptura de un contrato y herida narcisista : "Significaba el futuro para mí y así se ha llevado consigo todo futuro posible.” (Gay, 2006 : 422).

6 Durante el Siglo XIX, era común la conservación de mementos o reliquias del niño muerto -fotografías, un mechón de pelo-, práctica que ha sido analizada, para el caso de los Estados Unidos, como un modo de confirmar y reforzar la unión familiar y el afecto doméstico ante el advenimiento de una sociedad industrializada (Sanchez-Eppler, 2008: 109-114). En cambio, La vida de Dominguito, si bien constituye un memento del niño, no funciona como reafirmación de la familia, sino como puesta en escena de su crisis, así como de un legado paterno frustrado. Los lazos familiares aparecen como precarios, frágiles, permeados por la experiencia de la pérdida : es la falta del hijo la que pone en escena el amor y la afectividad paternas, y, asimismo, es el trabajo del duelo lo que refunda la identidad del padre.

7 De hecho, la figura de Sarmiento padre, que adopta a Dominguito y lo vuelve su heredero - "fue por adopción, cambiado su apellido por el de Sarmiento, que le fue nombrado tutor, a fin de que nada enfriase los afectos de la nueva familia" (Sarmiento, 1886:19)-y que, muerto el hijo, sufre desconsolado, señala un modelo de familia y de paternidad emergente en las dos últimas décadas del Siglo XIX en el Rio de la Plata. Se trata de un núcleo familiar reducido, de reproductividad contenida, ligado a fenómenos como el descenso de la mortalidad infantil, y la implementación de medidas de higiene y salud pública en el período (Moreno, 2004). Esta "nueva familia" de afectos, creada en base a lazos electivos, de adopción, y no exclusivamente sanguíneos o biológicos, como ha estudiado la crítica Carol Singley, responde a la transformación del modelo de familia patriarcal tradicional y lleva implícita la creencia en el progreso individual y colectivo, asociado al reformismo social y a la fe en el proceso de formación nacional (Singley, 2011 : 9-11). En La vida de Dominguito, la pérdida del hijo señala el estatuto precario de dicha "nueva familia" de afectos y pone en escena, como síntoma, la preocupación de Sarmiento sobre los posibles derroteros político-ideológicos de la Argentina futura, así como sobre las posibilidades de progreso individual y social.

8 La vida de Dominguito, cuya impronta autobiográfica ya ha sido señalada por la críticaSylvia Molloy afirma que el texto hace del hijo una copia de sí mismo y de su biografía una especie de autobiografía (Molloy, $1992: 144$ )-constituye un texto excepcional tanto en el corpus biográfico como autobiográfico de Sarmiento. De acuerdo a la lectura de Beatriz Sarlo y Carlos Altamirano, Recuerdos de Provincia establece un pasado nacional y un linaje de grandes hombres que culmina en el propio Sarmiento, cuya vida honraría su legado virtuoso (Sarlo y Altamirano, 1997 : 147). Asimismo, Sylvia Molloy ha leído en Recuerdos de Provincia la auto-representación de Sarmiento como paterfamilias, digno heredero de una cadena de antepasados ilustres (Molloy: 17). Sin embargo, Molloy advierte que se trata de un sujeto en crisis : crisis propia de la retórica de la auto-figuración, así como de la preocupación por la identidad nacional (Molloy : 9). En La vida de Dominguito, la muerte del 
hijo escenifica una interrupción de la genealogía, que provoca una crisis en la subjetividad del padre y funciona como síntoma de la dificultad de Sarmiento de concebir un legado político para la Argentina futura.

9 Los contextos histórico-políticos de La vida de Dominguito, en sus dos versiones, de 1867 y 1886, fueron para Sarmiento particularmente turbulentos. En el primer caso, el país se enfrentaba a su primer guerra moderna, la Guerra del Paraguay (1864-1870), que puso en evidencia el carácter todavía inestable del escenario político de la Argentina postindependentista y las dificultades para la organización nacional (Halperín Donghi, 1980 : 57). El segundo contexto, 1886 , fue aún más oscuro : aislado ideológica y políticamente de la generación gobernante, Sarmiento se encontraba desilusionado por los derroteros de la política nacional y por el accionar de unas clases ilustradas que estaban lejos de ofrecer el punto de partida para el proyecto político esperado (Halperín Donghi, 1994 : 30). La vida de Dominguito da cuenta de esta crisis del legado ideológico-político de Sarmiento, que emerge en el texto como una cesura en la relación entre el sujeto, el lenguaje y la mortalidad.

\section{Entre la primera y la última palabra}

La vida de Dominguito sigue las convenciones de la literatura elegíaca e incluye las últimas palabras de Dominguito, imbuidas de una carga poética específica de acuerdo a las reglas de un género preocupado por el poder y la finitud de la voz (Fuss, $2013: 12$ ). Sarmiento las cita de un cuaderno dedicado a la madre que el hijo dejó el día de la batalla en el bolsillo de su blusa :

Morir por su patria es vivir, es dar a nuestro nombre un brillo que nada borrará (...). Pero dejemos aquí estas líneas que un exceso de cariño me hace suponer ser letras póstumas que te dirijo (...). Son las diez. Las balas de grueso calibre estallan sobre el batallón : Salud mi madre! (Sarmiento, 1886 : 158).

El acto del soldado de dejar el cuaderno, cuando tenía presentimientos de muerte, en su ropa constituyó una práctica común en el campo de la batalla decimonónico : no sólo porque era un modo de garantizar la identificación del cadáver, sino también porque esas palabras le aseguraban a la madre-sujeto por antonomasia del duelo-que el soldado había tenido una buena muerte, una muerte patriótica, voluntaria y consentida (Gilpin Faust, 2008: 87 y 179). Sarmiento liga este cuaderno y estas palabras mortuorias con el cuaderno en que Dominguito aprendió a escribir, que contiene sus primeras palabras. Establece así un paralelo entre la muerte, clausura del discurso, y la escena infantil de acceso a la letra; muerte y nacimiento, origen y límite del lenguaje, representan dos momentos igualmente críticos, dos escenas de pasaje ligadas con la infancia. Escribe Giorgio Agamben :

Prueba palpable de la discontinuidad entre el mundo de los vivos y el mundo de los muertos, y entre sincronía y diacronía, y como significante inestable que puede transformarse en su opuesto todo el tiempo, el niño también representa al mismo tiempo una amenaza que se procura neutralizar y un expediente que hace posible el pasaje de una esfera a la otra sin abolir su diferencia significante. (Agamben, 2002, 90).

12 La infancia opera como significante flotante, móvil, que señala la diferencia y la discontinuidad temporal, la instancia del nacimiento y la finitud de la letra. Asimismo, Christopher Fynsk ha observado la fuerte ligazón entre la muerte del niño y la constitución del sujeto en el lenguaje: “...if we accept that the opening of language is 
indissociable from an experience (before experience) of a kind of death, there must be in our speaking, if only as a trace, the death of a child" (50).

La crítica ya ha advertido la centralidad de la escena de iniciación a la lectura en la escritura autobiográfica de Sarmiento, como primer ciudadano, futuro escritor y patriota (Molloy, 1992 : 18). En La vida de Dominguito, la escena de iniciación del hijo a la escritura adquiere una fuerza particular, desde la perspectiva de la pérdida y el duelo. Así, la palabra infantil se vuelve un puente hacia el pasado, invocación de una presencia fantasmal, así como reflexión sobre la relación a-genealógica, fuera del tiempo, del sujeto padre con el niño muerto. Con estas palabras describe Sarmiento el cuaderno :

El librito en blanco existe en poder de la madre y es guardado como una reliquia, pues que allí han quedado rastros indelebles del pasaje de una alma que se despierta y camina. Sería imposible dar idea de aquel prontuario, pues no hay sucesión de páginas o materias, y es una mezcla de sílabas formando palabras, figuras informes de geometría, desde las primeras páginas, un elefante aquí, más allá patos, garabatos que han querido explicar lo que las palabras dicen... (Sarmiento, 1886: 23)

14 La letra del niño funciona como un memento, una puerta al pasado y a su fantasma: Sarmiento llama la atención sobre el desorden de ese "prontuario"-su "mezcla de sílabas", "figuras informes" y "garabatos"-que, como puesta en crisis de la "sucesión" lineal del libro, escenifican el carácter liminal, imperfecto, de la letra infantil, el pasaje hacia la mudez del niño. Sarmiento se había referido a este cuaderno ya en la versión de 1867, como documento de la iniciación a la escritura :

Con tales ideas se presiente que teniendo un hijo, vivo, alegre, despierto inteligente este padre y maestro de lectura va a ensayar sus métodos de enseñar y realizar su idea de que los niños aprendan, lo mismo que juegan y corren y gritan. Pues bien así sucedió, y como si hubiera de dar cuenta un día del procedimiento, muerto temprana y gloriosamente el discípulo, le sobreviven las frágiles hojillas de papel en que aprendió a leer sin libros, sin silabario, sino conversando, jugando a leer, como se juega a correr carreras y a encumbrar la pandorga.

El discípulo tiene tres años y medio contados, es invierno hace frío y el maestro está sentado al lado de la chimenea, con el alumno al lado (...).

El alfabeto se fue animando poco a poco y el niño acabó por ser él mismo letra (...). Nuestro silabario era un librito en blanco. Ahí está : lo tengo a la vista ; fresco aún ; me parece que al abrirlo esta memoria exhala el perfume lechoso del niño de tres años ; él estaba a mi lado (Sarmiento, $2000: 27$ ).

Las "frágiles hojillas de papel", el "silabario", "librito en blanco" funcionan como marca del acceso del niño a la letra : esas hojas contienen al niño como una presencia fantasmal -“el niño acabó por ser él mismo letra"-que conversa, corre, grita y aprende a leer "sin libros", sin guión u orden previo, sino a partir del intercambio lúdico con el padre. En esas hojas, Sarmiento percibe el fantasma del niño de tres años y, a la vez, rememora su propia experiencia de padre y maestro : "es invierno hace frío y el maestro está sentado al lado de la chimenea", "Ahí está, lo tengo a la vista ; fresco aún; me parece que al abrirlo esta memoria exhala el perfume lechoso del niño de tres años; él estaba a mi lado". El niño, "muerto tempranamente", ha adquirido una realidad de discurso en el garabato, en las primeras letras, desordenadas, imperfectas, en las que Sarmiento lee "el perfume lechoso del niño de tres años", la presencia fantasmal de la infancia como pérdida. Escribe Sarmiento :

He aquí una muestra de lo que ha quedado escrito. Cuando ya hubo comprendido el fácil mecanismo, me dijo un día yo puedo escribir Sarmiento. -iQué has de escribir ! -Deme el carbón y verá. En dos páginas escribió lo que sigue litografiado: 
Sarmiento. La $\mathrm{S}$ está al revés pero es ese, la $\mathrm{m}$ es mayúscula pero la palabra está clara en la página, etc. (Sarmiento, 2000 : 27). escritura del nombre-que, demás está decir, coincide con el nombre del padre. La escritura imperfecta, fallida, de la primera palabra constituye un memento, reliquia de la infancia : el carácter abierto, en proceso, de la escritura de Dominguito funciona como un modo de invocar su fantasma. En la versión de 1886, la firma se incorpora en una reproducción litográfica :

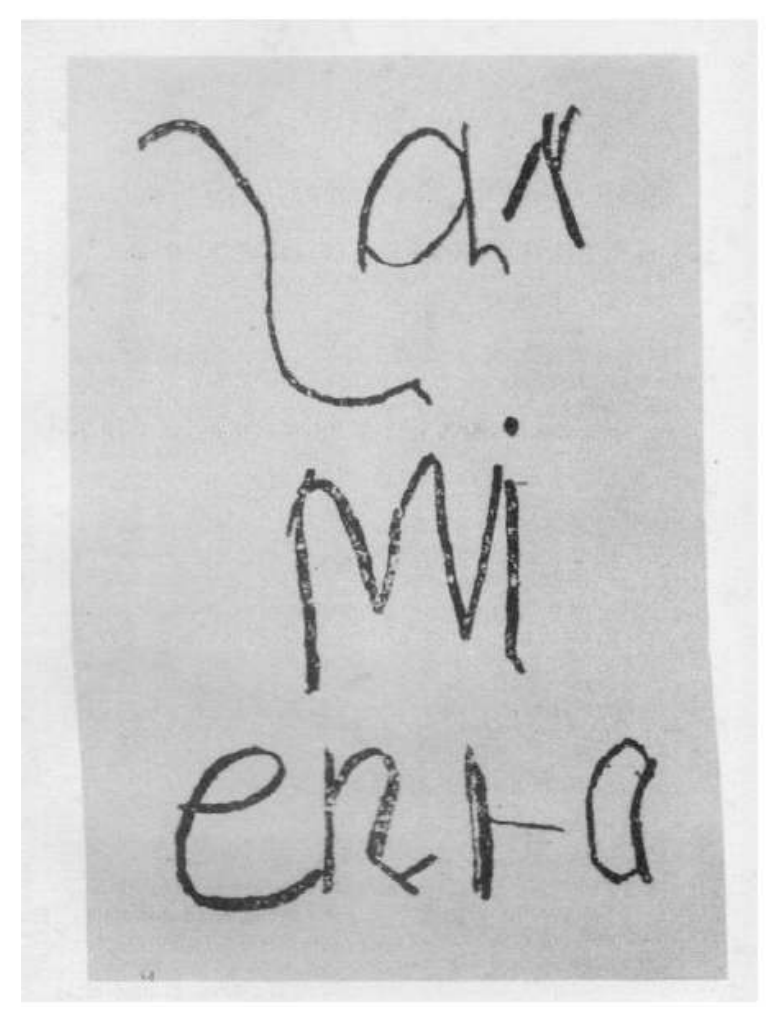

Figura 1 Sarmiento. La vida de Dominguito. In memoriam. 1886. P. 25.

La firma imperfecta de Dominguito pone en escena una instancia liminal, de frontera con el silencio de la muerte, a la vez que con la mudez de la infancia ; de este modo, la primera palabra hace hablar al niño muerto, doblemente mudo. Como sugiere Paul de Man, la figura de la prosopopeya, el discurso que se dirige y hace hablar a un ser ausente, fallecido o mudo, es un tropo central para la autobiografía :

...it is the figure of prosopopeia, the fiction of an apostrophe to an absent, deceased or voiceless entity, which posits the possibility of the latter's reply and confers upon it the power of speech. Voice assumes mouth, eye and finally face, a chain that is manifest in the etymology of the trope's name, prosopon poien, to confer a mask or a face (prosopon). Prosopopeia is the trope of the autobiography, by which one's name, as in the Milton poem, is made as intelligible and memorable as a face." (De Man, 926).

Sarmiento invoca al niño muerto y le confiere voz, rostro y máscara a través del garabato, la firma que lo vuelve inteligible y memorable. Al incorporar su escritura al propio texto, Sarmiento establece un contrato, un pacto mortuorio o fantasmal que vuelve indistinguibles las firmas y las identidades de ambos.

La primera palabra, de este modo, cumple una función análoga a la palabra póstuma en la poesía elegíaca: afirma un legado, sirve como memento y simboliza una dedicatoria 
postrera. Incorporada en el texto, la palabra "Sarmiento" señala una especie de pacto : designa al hijo como heredero del padre y al padre como heredero del hijo y, de este modo, reduplica el acto de adopción de Dominguito, con una nueva signatura del documento de paternidad, esta vez, firmada por el hijo, el descendiente. A través de la inclusión de la letra del niño, el recién llegado a la palabra, La vida de Dominguito explora la densidad poética del duelo. Sarmiento descubre la poesía a partir de la experiencia del dolor, como él mismo afirma :

A los cincuenta y seis años de una vida en que la lectura de todo llenó los intersticios que deja la acción sobre todo, leí por primera vez versos con amor, como si fueran los de Tennyson la expresión de mi propio dolor. Así concibo la poesía dando vueltas en torno de un pensamiento único, el dolor de una herida del corazón, la pérdida de un hijo.(Sarmiento, 2000 : 75)

Como se ve en la cita, la búsqueda poética resulta excepcional en una trayectoria ininterrumpida de compromiso político e intelectual ; la poesía emerge en la experiencia del duelo, a partir de la intensidad del afecto y del dolor desencadenados por la pérdida, así como de una fijación obsesiva al sujeto amado, que provoca un verdadero despertar del padre en Sarmiento.

\section{La paternidad como responsabilidad y supervivencia}

21 El psicoanálisis ha definido la noción de trauma alrededor de una paradoja: la imposibilidad de reaccionar o comprender del todo un evento violento inesperado o abrumador que retorna de modo repetitivo (Caruth, 1996 : 91-92). El trauma consiste en la simultánea necesidad e imposibilidad de responder, de modo tardío, a esa escena violenta que no se pudo aprehender, de la que se está constitutivamente ausente (Caruth, 1996 : 91-92). La crítica Cathy Caruth ha examinado el concepto del trauma a partir de un sueño, analizado por Freud y más tarde por Lacan, en que un padre oye a su hijo ya muerto, que le advierte que se está quemando. El padre se despierta y ve que, efectivamente, la persona que estaba al cuidado del cuerpo del niño se había quedado dormida y el niño yacía en llamas. Si bien no es el objetivo de este trabajo profundizar en el análisis del sueño, sí es importante señalar que el llamado del hijo determina la identidad del padre superviviente; su conciencia queda constituida por la imposibilidad de responder adecuadamente a ese llamado. Su despertar viene unido a un imperativo ético y una responsabilidad : el padre es aquel que debe decir lo doloroso e impensable, la mortalidad del hijo. Escribe Caruth: "It is precisely the dead child, the child in its irreducible inaccessibility and otherness, who says to the father: wake up, leave me, survive to tell the story of my burning" (105).

Sarmiento estuvo ausente en un sentido literal de la escena de la muerte del hijo, dado que sólo lo había visto por última vez cuatro años antes, en 1862, cuando Dominguito viajó a San Juan, donde el padre se desempeñaba como Gobernador (Anderson Imbert : 504). Estuvo ausente, también, de los ritos fúnebres celebrados en honor de los más de 2.000 soldados argentinos muertos en la batalla de Curupaití, ceremonia que unió al país en duelo (Leuchars, 2002: 153). Como si precisara remediar su ausencia de estas escenas, Sarmiento incorpora al texto una proliferante colección de documentos relativos a la vida y la muerte del hijo: necrologías aparecidas en los periódicos argentinos y chilenos, "Coronas fúnebres", los discursos dedicados a su muerte, una nota de pésame de la 
Universidad de Buenos Aires, testimonios de colegas de estudios y de compañeros de la batalla, un testimonio de su muerte, entre otros. El despertar de la paternidad en Sarmiento, como el padre que analiza Freud, se da a partir de una llamada o una invocación del hijo, que impone la necesidad y, a la vez, la imposibilidad, de narrar la escena de la que se estuvo ausente. La vida de Dominguito busca responder a esta necesidad, esta responsabilidad ética, del padre como sobreviviente, que se constituye en y por la invocación del hijo. Escribe Sarmiento :

Restaurado por el arte su imagen tal como yo la recordaba sobre la impresión fotográfica, logré una especie de resurrección, viviendo desde entonces con él, echándole una mirada al pasar, o deteniéndome a contemplarlo! Sonreírle, esperando locamente, y a veces creyendo que me sonreiría (...). ¿Por qué no ha de andar vagando en torno de lugares y objetos amados el genio, la sombra, el espíritu de los que se durmieron en el reposo eterno ? (...). Cuando mi dolor era más agudo, parecía oírle a lo lejos reír con aquel buen reír de los adolescentes para quienes la vida es un carnaval perenne? Cuando el tiempo empezaba a dulcificar aquella amargura creía oír el llanto del niño castigado reconviniendo entre cariñoso y ofendido por la severidad que siempre los de sus edad hallan infundada, y en uno u otro caso una consolación infinita inefable me traía estas formas singulares del recuerdo (Sarmiento, $2000: 76$ ).

En el momento más intenso del duelo, Sarmiento alucina la presencia del hijo que retorna, a su encuentro, en forma retardada: es el retrato animado que le sonríe, el espíritu que vaga, el adolescente que ríe y el niño pequeño que llora. El padre percibe su fantasma y su voz, como marcas de la otredad irreducible e inaccesible del hijo muerto. Hundido en un abismo de dolor, el padre halla consuelo ya no en el recuerdo estático del pasado, sino en el fantasma, imagen de su resurrección.

La vida de Dominguito invoca la presencia del hijo como fantasma, en su radical otredad, en un sentido profundamente ético. Como ha afirmado Diana Fuss, la poesía elegíaca, género de la consolación, en ocasiones puede resistirse a encontrar consuelo, en un gesto ético hacia los muertos (2013:7). En su alucinación, Sarmiento construye una instancia ética del desconsuelo, una ética del padre ante el fantasma del hijo. La vida de Dominguito es una reflexión sobre la infancia como escenificación de la instancia liminal del acceso al lenguaje, así como de la experiencia traumática de la muerte filial, que provoca un despertar de la subjetividad del padre y lo interpela en un sentido ético.

\section{BIBLIOGRAFÍA}

Agamben, Giorgio. Infancia e historia. Ensayo sobre la destrucción de la experiencia. Madrid : Editora Nacional, 2002

Anderson Imbert, Enrique. "Génesis del primer Dominguito" Nueva Revista de Filología Hispánica 24. 2 (1975).

Caruth, Cathy. Unclaimed Experience : Trauma, narrative and history. Baltimore : John Hopkins Univ. Press, 1996. 
Derrida, Jacques. The Postcard. From Socrates to Freud and Beyond. Chicago : Chicago University Press, 1987.

Fariña Nuñez, Porfirio. Los Amores de Sarmiento. Buenos Aires : Tor, 1935.

Freud, Sigmund. "Mourning and Melancholia." The Standard Edition of the Complete Psychological Works of Sigmund Freud. vol. XIV. London : The Hogharth Press, 1973.

Freud, Sigmund. Beyond the Pleasure Principle. New York : Norton, 1961.

Fuss, Diana. Dying Modern. A Meditation on elegy. Durham : Duke UP, 2013.

Fynsk, Christopher. Infant Figures. The Death of the Infans and Other Scenes of Origin. California : Stanford UP, 2000.

Gay, Peter. Freud. A life for our time. New York : W.W. Norton, 2006.

Gilpin Faust, Drew. This Republic of Suffering. Death and the American Civil War. New York : Knopf, 2008.

Halperín Donghi. Tulio. “Una nación para el desierto Argentino.”, Tulio Halperín Donghi (ed.). Proyecto de construcción de una nación. Argentina : 1846-1880. Caracas : Biblioteca Ayacucho, 1980.

Leuchars, Christopher. To The Bitter End. Paraguay and the War of the Triple Alliance. London : Greenwood Press, 2002.

Molloy, Sylvia. At Face Value. Autobiographical Writing in Spanish America. Cambridge : Cambridge UP, 1992.

Moreno, José Luis. Historia de la familia en el Río de la Plata. Buenos Aires : Sudamericana, 2004.

Singley, Carol. Adopting America. Childhood, Kinship, and National Identity in Literature. Oxford :

Oxford University Press, 2011.

Sarlo, Beatriz y Carlos Altamirano. "Una vida ejemplar : La estrategia de Recuerdos de Provincia” Ensayos argentinos. De Sarmiento a la vanguardia. Buenos Aires : Ariel, 1997.

Sarmiento, Domingo Faustino. La vida de Dominguito. Pról. de Javier Fernández. Buenos Aires : Fondo Nacional de las Artes, 2000.

\section{NOTAS}

1. Existen dos versiones del texto: la primera versión, una serie de apuntes escritos un año después de fallecido Dominguito, en 1867 en Washington, continuó inédita, como parte de los archivos del Museo Histórico Sarmiento hasta el año 2000, cuando fue publicada por el Fondo Nacional de las Artes, con el título de La vida de Dominguito. La segunda versión, con el extenso título de La vida de Dominguito. In memoriam del valiente y deplorado capitán Domingo Fidel Sarmiento. Muerto en Curupaití a los veinte años de edad. Autor de varios escritos, biografías y correspondencias y traductor de "París en América", fue publicada por la Sociedad Tipográfica "El Censor" en 1886, dos años antes del fallecimiento de Sarmiento, con motivo del vigésimo aniversario de la muerte del hijo. A partir de aquí, cito la versión de 1867 como La vida de Dominguito y la de 1886 como La vida de Dominguito. In memoriam.

2. Porfirio Fariña Nuñez, en Los Amores de Sarmiento (Buenos Aires: Tor, 1935) discute extensamente la "dudosa" filiación de Dominguito, y opta por atribuir la paternidad biológica a Sarmiento. En “Génesis del primer Dominguito" (Nueva Revista de Filología Hispánica 24. 2 Homenaje a Raimundo Lida (1975) : 504-9), ensayo sobre la primera versión del texto, Anderson Imbert expone una hipótesis similar. 
3. Para una lectura del poema de Mallarmé, consultar Fynsk, Christopher, Infant Figures. The Death of the Infans and Other Scenes of Origin. California : Stanford UP, 2000. P. 86-87.

\section{RESÚMENES}

Este ensayo reflexiona sobre la escena de la muerte del niño en La vida de Dominguito, de Domingo Faustino Sarmiento en un sentido doble : por un lado, en su relación con el lenguaje, como evento que pone en escena el acceso a la letra $y$, por otro, como trauma, que establece una discontinuidad fundamental en la experiencia del padre. Se postula que la infancia aparece en el texto en tres niveles : en primer lugar, el niño rememorado en la escritura autobiográfica; en segundo, la letra del hijo incorporada en el texto; y por último, la muerte del niño como emergencia del trauma en su irreducible otredad. Se concluye que dicha escena plantea una necesidad ética a la que el texto busca responder.

Cet essai propose une réflexion sur la scène de la mort de l'enfant dans La vida de Dominguito de Domingo Faustino Sarmiento à partir de deux aspects: sa relation avec le langage et son caractère traumatique. D'un côté, la mort de l'enfant est envisagée comme l'événement mettant en scène l'accès à l'écriture, de l'autre, comme un traumatisme qui établit une discontinuité fondamentale dans l'expérience du père. L'enfance apparaît dans le texte à trois niveaux : comme l'objet du souvenir dans l'écriture autobiographique; comme l'écriture infantile intégrée au texte et enfin à travers la mort de l'enfant comme manifestation du traumatisme dans son irréductible étrangeté. On en conclut que cette scène pose un problème éthique auquel le texte cherche à répondre.

This essay analyzes the figure of the death of the child in La vida de Dominguito, by Domingo Faustino Sarmiento, in a double sense : on the one hand, in its relationship with language, as an event that symbolizes the coming to the word and, on the other, as a trauma that establishes a fundamental discontinuity in the father's experience. It examines the emergence of childhood at three different levels : first, the child as remembered through autobiographical writing; second, the child's written word as incorporated to the text; and last, the death of the child as a traumatic experience in its irreducible otherness. It concludes that such scene poses an ethical necessity to which the text seeks to respond.

\section{ÍNDICE}

Mots-clés: enfance, écriture, traumatisme, Domingo Faustino Sarmiento

Palabras claves: infancia, escritura, trauma, Domingo Faustino Sarmiento

Keywords: childhood, writing, trauma, Domingo Faustino Sarmiento 
AUTOR

ALEJANDRA JOSIOWICZ

Assistant Professor

Rutgers University 